\title{
Risk Factors for Postoperative Ileus Following Orthopedic Surgery: The Role of Chronic Constipation
}

\author{
Tae Hee Lee, ${ }^{1,2}$ Joon Seong Lee, ${ }^{2 *}$ Su Jin Hong, ${ }^{2}$ Jae Young Jang, ${ }^{1,2}$ Seong Ran Jeon, ${ }^{1,2}$ Dong Won Byun, ${ }^{1}$ Won Young Park, ${ }^{2}$ \\ Soon Im Kim, ${ }^{3}$ Hyung Suk Choi, ${ }^{4}$ Jae Chul Lee, and Ji Sung Lee ${ }^{5}$ \\ ${ }^{1}$ Nutritional Support Team, Soonchunhyang University Seoul Hospital, Seoul, Korea; ${ }^{2}$ Institute for Digestive Research, Soonchunhyang \\ University, College of Medicine, Seoul, Korea; Departments of ${ }^{3}$ Anesthesiology and Pain Medicine, and ${ }^{4}$ Orthopedic Surgery, Soonchunhyang \\ University, College of Medicine, Seoul, Korea; and ${ }^{5}$ Biostatistical Consulting Unit, Soonchunhyang University Medical Center, Seoul, Korea
}

\section{Background/Aims}

Distinction is vague between severe constipation and postoperative ileus (POI) in terms of pathogenesis, clinical features, and treatment options. However, no data are available regarding their associations.

\section{Methods}

After retrospective review of data from patients who underwent orthopedic surgery during the first 6 months of 2011, a total of 612 patients were included. Severe constipation was defined as symptoms of constipation requiring treatment using at least 2 laxatives from different classes for at least 6 months. POI was defined as paralytic ileus lasting more than 3 days post-surgery and associated with 2 or more of the following: (1) nausea/vomiting, (2) inability to tolerate an oral diet over a 24-hour period, and (3) absence of flatus over a 24-hour period. The subjects were divided into non-POI and POI groups, and we compared patient-, surgery-, and pharmaceutical-related factors.

\section{Results}

Thirteen (2.1\%) out of 612 experienced POI. In comparisons between the non-POI and POI groups, univariate analysis showed significant differences in the mean age (51.4 vs 71.6 years), mean body mass index $\left(24.1 \mathrm{vs} 21.8 \mathrm{~kg} / \mathrm{m}^{2}\right)$, severe constipation $(5.8 \%$ vs $76.9 \%$ ), co-morbidities (33.2\% vs $84.6 \%$ ), type of orthopedic surgery (spine/hip/limb: $19.4 / 11.0 / 65.6 \%$ vs $23.1 / 61.5 / 15.4 \%$ ), and estimated blood loss (50 vs $300 \mathrm{~mL}$ ). Multivariate logistic regression analysis, after adjustment for age, body mass index, co-morbidities, type of orthopedic surgery, and estimated blood loss, showed that severe constipation was an independent risk factor for POI (OR, 35.23; 95\% Cl, 7.72-160.82; $P<0.001)$.

\section{Conclusions}

Severe constipation is associated with POI after orthopedic surgery.

(J Neurogastroenterol Motil 2015;21:121-125)

Key Words

lleus; Orthopedics; Risk factors

Received: July 2, 2014 Revised: August 13, 2014 Accepted: August 15, 2014

(c) This is an Open Access article distributed under the terms of the Creative Commons Attribution Non-Commercial License (http://creativecommons. org/licenses/by-nc/3.0) which permits unrestricted non-commercial use, distribution, and reproduction in any medium, provided the original work is properly cited.

*Correspondence: Joon Seong Lee, PhD, MD Institute for Digestive Research, Soonchunhyang University, College of Medicine, Deasagwan-gil 22, Yongsan-gu, Seoul 140-743, Korea

Tel: +82-2-709-9691, Fax: +82-2-709-9696, E-mail: joonlee@schmc.ac.kr

Tae Hee Lee and Joon Seong Lee contributed equally to this work.

Financial support: This work was supported by the Soonchunhyang University Research Fund.

Conflicts of interest: None.

Author contributions: Tae Hee Lee, Joon Seong Lee, Su Jin Hong, Jae Young Jang, Seong Ran Jeon, and Won Young Park collected data and drafted the paper; Soon Im Kim edited the paper; Dong Won Byun, Hyung Suk Choi, and Jae Chul Lee reviewed data analysis; and Ji Sung Lee performed statistical analyses.

ORCID: Tae Hee Lee, http://orcid.org/0000-0003-3049-8252. 


\section{Introduction}

Postoperative ileus (POI) is defined as temporarily impaired gastrointestinal motility following surgery. The adverse effects of POI include increased postoperative pain, nausea and vomiting, delay in enteral nutrition, poor wound healing, delay in postoperative mobilization, increased risk of other postoperative complications, prolonged hospitalization, decreased patient satisfaction, and increased health care costs. ${ }^{1}$ Although the incidence of POI is highest for intra-abdominal surgery cases, it can also develop after orthopedic surgery. The incidence of POI after lower extremity reconstruction ranges from $0.3 \%$ to $2.0 \%{ }^{2-4}$ with an even higher incidence $(5.6 \%)$ following revision total hip arthroplasty. ${ }^{5}$

Colonic dysfunction is the most critical factor limiting the resolution of POI. ${ }^{6}$ Dysmotility of the colon is the cause of slow transit constipation. ${ }^{7,8}$ Despite a lack of strong evidence for their clinical utility, laxatives or prokinetics have been used to treat POI in clinical practice. There are similarities in the pathogenesis, clinical features, and treatment options between severe constipation and POI. In this context, the relationship between severe constipation and POI needs to be elucidated. This study aimed to evaluate severe constipation as a potential independent risk factor for POI in patients undergoing orthopedic surgery.

\section{Materials and Methods}

Consecutive patients $(n=677)$ who had undergone orthopedic surgery during the first 6 months of 2011 were reviewed retrospectively. Those patients with no available data on their postoperative outcomes $(\mathrm{n}=9)$, who underwent repeated orthopedic surgery within the same period $(n=13)$, or who underwent concurrent bowel, brain or thorax surgery $(n=43)$ were excluded. After applying these exclusion criteria, 612 patients were included in the study. Most of the patients were administered a standard anesthesia regimen, consisting of $2 \mathrm{mg} / \mathrm{kg}$ propofol, $20 \mu \mathrm{g} / \mathrm{min}$ remifentanil infusion, and $0.6 \mathrm{mg} / \mathrm{kg}$ rocuronium. Anesthesia was maintained using desflurane, 50\% nitrous oxide and $5-20 \mu \mathrm{g} / \mathrm{min}$ remifentanil infusion. The patients also received $0.2-\mathrm{mg}$ glycopyrrolate intramuscularly as premedication 30 minutes prior to surgery. Approximately $10 \mathrm{mi}-$ nutes before finishing surgery, a 50-100 $\mu \mathrm{g}$ bolus dose of fentanyl and 30-mg ketorolac were administered intravenously. Simultaneously, 8-mg ondansetron was injected intravenously to prevent postoperative nausea and vomiting. For postoperative pain control, $523(85.5 \%)$ patients received intravenous patient-controlled analgesia (PCA) using a bolus dose of $15-\mu \mathrm{g}$ fentanyl, a lockout interval of 5 minutes, and no basal infusion for 48 hours after orthopedic surgery. All patients were managed using a similar postoperative management plan. Nasogastric tubes were not used routinely unless there was a significant amount of postoperative nausea, vomiting, and/or abdominal distention. All patients were offered a clear liquid diet orally on postoperative day 1 and were progressively advanced to a solid diet as tolerated. The study protocol was approved by the Institutional Review Board of Soonchunhyang University Seoul Hospital, College of Medicine, Seoul, Korea.

\section{Data Collection}

We evaluated the following variables: (1) patient-related factors (age, gender, body mass index, severe constipation, and co-morbidities); (2) surgery-related factors (type of anesthesia; ie, general vs spinal vs brachial plexus block; type of orthopedic surgery; ie, spine vs hip vs limb surgery; operative time; and estimated blood loss; and (3) pharmaceutical-related factors (use of opioids or PCA). The definition of severe constipation was based on all of the following: (1) onset of constipation symptoms at least 6 months prior to orthopedic surgery, (2) previous treatment with at least 2 laxatives from different classes for at least 6 months, and (3) no evidence of organic diseases causing constipation. POI was defined as paralytic ileus lasting more than 3 days post-surgery and associated with two or more of the following: (1) nausea/vomiting, (2) inability to tolerate an oral diet over a 24-hour period, and (3) absence of flatus over 24-hour period.

\section{Statistical Methods}

A preliminary analysis using Fisher's exact test was conducted to determine if there were any differences in severe constipation between patients with (POI group) and without POI (non-POI group). The patient-, surgery-, and pharmaceuticalrelated factors were also compared between the POI and nonPOI groups using the Student's $t$ test, Wilcoxon rank sum test, Chi-square test, or Fisher's exact test.

Multivariate logistic regression analysis, after adjusting for significant variables determined from the univariate analyses, was performed to assess the adjusted OR for constipation in the POI group. 


\section{Results}

POI was documented in $13(2.1 \%)$ out of the 612 patients. Table 1 shows the comparisons between the POI and non-POI groups. Patients in the POI group were significantly older (mean age $71.6 \pm 19.3$ years) than those in the non-POI group (mean age $51.4 \pm 20$ years $)(P<0.001)$. BMI was significantly lower in the POI group $\left(21.8 \pm 5.1 \mathrm{~kg} / \mathrm{m}^{2}\right)$ compared with the non-POI group $\left(24.1 \pm 3.7 \mathrm{~kg} / \mathrm{m}^{2}\right)(P=0.025)$. There was no significant gender difference between the 2 groups. Ten (76.92\%) patients in the POI group had severe constipation, while 35 (5.84\%) did in the non-POI group, revealing a significantly higher proportion in the POI group $(P<0.001)$. Co-morbidities were significantly different between the 2 groups, in which the POI group had a significantly higher proportion of patients with hypertension, diabetes, liver disease, and miscellaneous diseases. The 2 groups were comparable in terms of the proportion of patients with a previous history of abdominal surgery. Spine, hip, and limb surgeries were performed in 3 (23.08\%), 8 $(61.54 \%)$, and $2(15.38 \%)$ patients in the POI group and in 116 (19.40\%), $66(11.04 \%)$, and $416(69.57 \%)$ patients in the non-POI group, respectively. There were significant differences in the type of orthopedic surgery $(P<0.001)$, while the operation times were comparable between the 2 groups. The POI group had a significantly higher estimated blood loss compared with the non-POI group $(P=0.001)$. However, the proportion of patients managed with PCA or postoperative opioids did not differ between the 2 groups. After adjusting for age, body mass index, hypertension, diabetes, miscellaneous diseases, type of or-

Table 1. Comparison of Patients With and Without Postoperative Ileus

\begin{tabular}{|c|c|c|c|}
\hline & Non-POI $(\mathrm{n}=599)$ & POI $(\mathrm{n}=13)$ & $P$-value \\
\hline Age (mean $\pm \mathrm{SD}, \mathrm{yr})$ & $51.4 \pm 20.0$ & $71.6 \pm 19.3$ & $<0.001^{\mathrm{a}}$ \\
\hline$<65(\mathrm{n}[\%])$ & $420(70.12)$ & $4(30.77)$ & $0.004^{\mathrm{b}}$ \\
\hline$\geq 65(\mathrm{n}[\%])$ & $179(29.88)$ & $9(69.23)$ & \\
\hline Body mass index $\left(\right.$ mean $\left.\pm \mathrm{SD}, \mathrm{kg} / \mathrm{m}^{2}\right)$ & $24.1 \pm 3.7$ & $21.8 \pm 5.1$ & $0.025^{\mathrm{a}}$ \\
\hline Gender (n [\%]) & & & $0.381^{\mathrm{c}}$ \\
\hline Male & $304(50.75)$ & $5(38.46)$ & \\
\hline Female & $295(49.25)$ & $8(61.54)$ & \\
\hline Severe constipation (n [\%]) & $35(5.84)$ & $10(76.92)$ & $<0.001^{\mathrm{b}}$ \\
\hline Comorbidities (n [\%]) & $199(33.22)$ & $11(84.62)$ & $0.001^{\mathrm{b}}$ \\
\hline Hypertension & $134(22.37)$ & $10(76.92)$ & $0.001^{\mathrm{b}}$ \\
\hline Diabetes & $70(11.69)$ & $7(53.85)$ & $0.001^{\mathrm{b}}$ \\
\hline Liver diseases & $17(2.84)$ & $2(15.38)$ & $0.058^{\mathrm{b}}$ \\
\hline Tuberculosis & $7(1.17)$ & $0(0.00)$ & $>0.999^{\mathrm{b}}$ \\
\hline Kidney diseases & $10(1.67)$ & $1(7.69)$ & $0.212^{\mathrm{b}}$ \\
\hline Cardiovascular diseases & $27(4.51)$ & $2(15.38)$ & $0.122^{\mathrm{b}}$ \\
\hline Lung diseases & $13(2.17)$ & $1(7.69)$ & $0.262^{\mathrm{b}}$ \\
\hline Miscellaneous & $29(4.84)$ & $3(23.08)$ & $0.026^{\mathrm{b}}$ \\
\hline History of abdominal surgery (n [\%]) & $19(3.17)$ & $1(7.69)$ & $0.354^{\mathrm{b}}$ \\
\hline Types of orthopedic surgery (n [\%]) & & & $<0.001^{\mathrm{b}}$ \\
\hline Spine & $116(19.40)$ & $3(23.08)$ & \\
\hline Hip & $66(11.04)$ & $8(61.54)$ & \\
\hline Limb & $416(69.57)$ & $2(15.38)$ & \\
\hline Types of anesthesia (n [\%]) & & & $0.604^{\mathrm{b}}$ \\
\hline General & $459(76.63)$ & $9(69.23)$ & \\
\hline Spinal & $129(21.54)$ & $4(30.77)$ & \\
\hline Brachial plexus block & $11(1.84)$ & $0(0.00)$ & \\
\hline Operation time (median [IQR]) & $90(55-140)$ & $90(60-140)$ & $0.531^{\mathrm{d}}$ \\
\hline Estimated blood loss (median [IQR]) & $50(10-300)$ & $300(200-400)$ & $0.001^{\mathrm{d}}$ \\
\hline Patient-controlled analgesia (n [\%]) & $511(85.31)$ & $12(92.31)$ & $0.704^{\mathrm{b}}$ \\
\hline Postoperative opioid (n [\%]) & $523(87.31)$ & $13(100.00)$ & $0.387^{\mathrm{b}}$ \\
\hline
\end{tabular}

POI, postoperative ileus; IQR, interquartile range.

${ }^{\mathrm{a}} P$-value by Student's $t$ test, ${ }^{\mathrm{b}} P$-value by Chi-square test, ${ }^{\mathrm{c}} P$-value by Fisher's exact test, ${ }^{\mathrm{d}} P$-value by Wilcoxon rank sum test. 
Table 2. The Adjusted Odds Ratio for Severe Constipation in Postoperative Ileus

\begin{tabular}{cccc}
\hline & Adjusted OR & $95 \% \mathrm{CI}$ & $P$-value \\
\hline Severe constipation & 35.23 & $7.72-160.82$ & $<0.001$ \\
\hline
\end{tabular}

Adjusting for age, body mass index, hypertension, diabetes, miscellaneous diseases, type of orthopedic surgery, and estimated blood loss.

thopedic surgery and estimated blood loss, multiple logistic regression analysis showed that severe constipation was an independent risk factor for POI (OR, 35.23; 95\% CI, 7.72160.82; $P<0.001$ ) (Table 2). Aside from severe constipation, independent risk factors of POI were age and type of orthopedic surgery. With regard to POI, those aged $<65$ years were $85 \%$ less likely to develop POI than those aged $\geq 65$ years (OR, 0.15 ; 95\% CI, 0.02-0.95; $P=0.044)$, after adjusting for severe constipation, body mass index, hypertension, diabetes, miscellaneous diseases, type of orthopedic surgery, and estimated blood loss. A significant difference in POI was found between those who underwent spine and limb surgery $(P=0.047)$. Those who underwent limb surgery were $90 \%$ less likely to develop POI than those who underwent spine surgery (OR, 0.10; 95\% CI, 0.01-0.97), after adjusting for age, severe constipation, body mass index, hypertension, diabetes, miscellaneous diseases, and estimated blood loss.

\section{Discussion}

This is the first study to show that severe constipation is significantly associated with POI following orthopedic surgery. Our results support those of an earlier study, which reported that the use of an effective bowel protocol decreased constipation and POI in patients undergoing orthopedic surgery. ${ }^{9}$ In that study, a new bowel program consisting of both a stool softener and routine use of a bisacodyl suppository on postoperative day 1 significantly decreased the rate of POI from 26.67 to 0.0 per 1,000 cases $(P=0.123)$ in patients with hip arthroplasty. The rate of constipation also decreased significantly from 120.0 to 37.04 per 1,000 cases $(P=0.001)$.

The mechanism underlying this association remains unknown but may be based largely on the similar proposed mechanisms for both severe constipation and POI. Many studies have also attempted to identify the causes of slow transit constipation, such as autonomic nervous dysfunction, enteric nervous dysfunction, and neuroendocrine dysfunction. ${ }^{10-16}$ These mechanisms are also presumed to be involved in the development of POI. ${ }^{1}$ In particular, neural and hormonal factors (eg, nitric oxide, vasoactive intestinal peptide, and substance $\mathrm{P}$ ) participate in normal gastrointestinal motility, constipation, and POI.

An association between severe constipation and POI is also supported by their similar pharmacologic interventions. One non-randomized study evaluated 20 consecutive patients took laxatives postoperatively and reported reduced times to flatus and the first bowel movement, as well as decreased hospitalization stays, compared with historical controls. ${ }^{17}$ 5-hydroxytryptamine receptor 4 agonists, such as cisapride, mosapride and prucalopride, are potent prokinetic agents that exert effects on the upper and lower gastrointestinal tract. ${ }^{18}$ Importantly, these medications are used to treat POI as well as chronic constipation, even though cisapride was withdrawn from the market for cardiovascular safety reasons. ${ }^{19-21}$ The highly selective 5 -hydroxytryptamine receptor 4 agonist, prucalopride, has been indicated for the treatment of chronic constipation, especially in patients who do not experience adequate relief with laxatives. ${ }^{22}$ In a phase II trial for POI, 317 patients who underwent partial colectomy were administered 0.5-, 2-, or 4-mg prucalopride once daily until the third day post-surgery. Compared with the placebo group, the 4-mg prucalopride group showed a faster gastrointestinal motility recovery time and a $10 \%$ increase in the number of patients released from the hospital within 6 days. ${ }^{23}$ This study has limitations, mostly stemming from its retrospective design.

As with all retrospective studies, the present study was limited in its ability to determine cause-effect relationships and to control for all possible confounders. There was no significant difference in the proportion of patients who received PCA or postoperative opioids. This did not mean that the total amount of opioids used was comparable between the 2 groups. Limiting opiate use in the postoperative setting has been associated with a significant decrease in the duration of POI. ${ }^{24}$ Although we did not assess the exact dose of postoperative opioids consumed, it was expected to be lower in the POI patients. This was because the POI diagnosis was made by gastroenterologists and because therapeutic interventions for POI, including a greater use of nonsteroidal anti-inflammatory drugs but lesser use of opiates for pain control, were actively performed.

The highly adjusted OR for severe constipation might result from very low probability events for POI. POI occurs most commonly in patients who have undergone abdominal surgery. However, manipulation of the bowel affects postoperative inflammatory responses in the intestinal muscularis, thereby pro- 
longing the POI. ${ }^{1}$ Our study population appeared to be appropriate for decreasing the potential for confounding occurrences (eg, bowel manipulation or inflammatory mediation effects).

To date, various procedures and agents - including laparoscopic surgery, thoracic epidurals, nonsteroidal anti-inflammatory drugs, and opiate antagonists, early feeding and ambulation, laxatives, and possibly prokinetics - have shown clinical benefit in some. In our study, the adjusted odds ratio for POI development in patients with severe constipation was 35.23. This suggests that pharmacologic interventions for severe constipation might be added to the list of interventions efficacious for POI. We believe that our study will stimulate further research in this area. Gastroenterologists could make important contributions to the effort to decrease POI in orthopedic surgery patients with severe constipation.

\section{References}

1. Behm B, Stollman N. Postoperative ileus: etiologies and interventions. Clin Gastroenterol Hepatol 2003;1:71-80.

2. Bederman SS, Betsy M, Winiarsky R, Seldes RM, Sharrock NE, Sculco TP. Postoperative ileus in the lower extremity arthroplasty patient. J Arthroplasty 2001;16:1066-1070.

3. Pavone V, Johnson T, Saulog PS, Sculco TP, Bottner F. Perioperative morbidity in bilateral one-stage total knee replacements. Clin Orthop Relat Res 2004;421:155-161.

4. Nelson JD, Urban JA, Salsbury TL, Lowry JK, Garvin KL. Acute colonic pseudo-obstruction (Ogilvie syndrome) after arthroplasty in the lower extremity. J Bone Joint Surg Am 2006;88:604-610.

5. Berend KR, Lombardi AV Jr, Mallory TH, Dodds KL, Adams JB. Ileus following total hip or knee arthroplasty is associated with increased risk of deep venous thrombosis and pulmonary embolism. J Arthroplasty 2004;19(suppl 2):82-86.

6. Woods JH, Erickson LW, Condon RE, Schulte WJ, Sillin LF. Postoperative ileus: a colonic problem? Surgery 1978;84:527-533.

7. Burleigh DE. Evidence for a functional cholinergic deficit in human colonic tissue resected for constipation. J Pharm Pharmacol 1988;40: 55-57.

8. Bassotti G, Chiarioni G, Imbimbo BP, et al. Impaired colonic motor response to cholinergic stimulation in patients with severe chronic idiopathic (slow transit type) constipation. Dig Dis Sci 1993;38: 1040-1045.

9. Linari LR, Schofield LC, Horrom KA. Implementing a bowel program: is a bowel program an effective way of preventing constipation and ileus following elective hip and knee arthroplasty surgery?
Orthop Nurs 2011;30:317-321

10. Roe AM, Bartolo DC, Mortensen NJ. Slow transit constipation. Comparison between patients with or without previous hysterectomy. Dig Dis Sci 1988;33:1159-1163.

11. Tomita R, Fujisaki S, Ikeda T, Fukuzawa M. Role of nitric oxide in the colon of patients with slow-transit constipation. Dis Colon Rectum 2002;45:593-600.

12. Tomita R, Tanjoh K, Fujisaki S, Ikeda T, Fukuzawa M. Regulation of the enteric nervous system in the colon of patients with slow transit constipation. Hepatogastroenterology 2002;49:1540-1544.

13. Cortesini C, Cianchi F, Infantino A, Lise M. Nitric oxide synthase and VIP distribution in enteric nervous system in idiopathic chronic constipation. Dig Dis Sci 1995;40:2450-2455.

14. Milner P, Crowe R, Kamm MA, Lennard-Jones JE, Burnstock G. Vasoactive intestinal polypeptide levels in sigmoid colon in idiopathic constipation and diverticular disease. Gastroenterology 1990;99: 666-675.

15. Porter AJ, Wattchow DA, Hunter A, Costa M. Abnormalities of nerve fibers in the circular muscle of patients with slow transit constipation. Int J Colorectal Dis 1998;13:208-216.

16. Tzavella K, Riepl RL, Klauser AG, Voderholzer WA, Schindlbeck NE, Muller-Lissner SA. Decreased substance P levels in rectal biopsies from patients with slow transit constipation. Eur J Gastroenterol Hepatol 1996;8:1207-1211.

17. Fanning J, Yu-Brekke S. Prospective trial of aggressive postoperative bowel stimulation following radical hysterectomy. Gynecol Oncol 1999;73:412-414.

18. Gershon MD. Review article: serotonin receptors and transporters -roles in normal and abnormal gastrointestinal motility. Aliment Pharmacol Ther 2004;20(suppl 7):3-14.

19. Toyomasu Y, Mochiki E, Morita H, et al. Mosapride citrate improves postoperative ileus of patients with colectomy. J Gastrointest Surg 2011;15:1361-1367.

20. Narita K, Tsunoda A, Takenaka K, Watanabe M, Nakao K, Kusano $\mathrm{M}$. Effect of mosapride on recovery of intestinal motility after handassisted laparoscopic colectomy for carcinoma. Dis Colon Rectum 2008;51:1692-1695.

21. Zeinali F, Stulberg JJ, Delaney CP. Pharmacological management of postoperative ileus. Can J Surg 2009;52:153-157.

22. Eswaran S, Guentner A, Chey WD. Emerging pharmacologic therapies for constipation-predominant irritable bowel syndrome and chronic constipation. J Neurogastroenterol Motil 2014;20:141-151.

23. Galandiuk S, Beyens G, Ausma J, Vandeplassche L. Evaluation of the efficacy, safety and tolerability of prucalopride (Resolor $\left.{ }^{\circledR}\right)$ given subcutaneously in patients undergoing elective partial colectomies. Gastroenterology 2008;134(suppl 1):A-138.

24. Ferraz AA, Cowles VE, Condon RE, et al. Nonopioid analgesics shorten the duration of postoperative ileus. Am Surg 1995;61:10791083. 